\title{
ComposiçÃo Química da Cera Epicuticular de Biótipos de Azevém Resistente e Suscetível ao Glyphosate ${ }^{1}$
}

\author{
Chemical Composition of the Epicuticular Wax of Italian Ryegrass Biotypes Resistant and \\ Susceptible to Glyphosate
}

\author{
GUIMARÃES, A.A. ${ }^{2}$, FERREIRA, E.A. ${ }^{3}$, VARGAS, L. ${ }^{4}$, SILVA, A.A. ${ }^{5}$, VIANA, R.G. ${ }^{6}$, DEMUNER, A.J. ${ }^{7}$, \\ CONCENÇO, G. ${ }^{6}$, ASPIAZU, I. ${ }^{6}$, GALON, L. ${ }^{3}$, REIS, M.R. ${ }^{6}$ e SILVA, A.F. ${ }^{6}$
}

\begin{abstract}
RESUMO - Objetivou-se com este trabalho determinar a composição química da cera epicuticular dos biótipos de azevém (Lolium multiflorum) resistente e suscetivel ao glyphosate, buscando relações entre suas características e a resistência dos biótipos ao herbicida. A cera epicuticular foi extraida e quantificada e os seus constituintes analisados por cromatografia em fase gasosa, acoplada a espectrômetro de massa (CG-EM). Para determinação da composição química, amostras de lâmina foliar foram retiradas 30 dias após a emergência das plantas, coletando-se a primeira folha com ligula totalmente visivel. A quantidade de cera epicuticular extraída não diferiu entre os biótipos. Entre os compostos que constituem a cera epicuticular, os álcoois são os mais abundantes, sendo representados por apenas um composto: o hexacosan-1-ol (46,80\% no biótipo resistente e 52,20\% no biótipo suscetivel). Ao comparar a polaridade da cera epicuticular dos biótipos de azevém, constatou-se que tanto no biótipo resistente quanto no suscetivel a cera epicuticular apresentou mais de 50\% de componentes polares (álcoois e aldeídos) em sua constituição, sendo esse valor igual a $69,80 \%$ no biótipo resistente e $64,94 \%$ no biótipo suscetivel. Por meio da caracterização apresentada, pode-se afirmar que existem pequenas diferenças na cera epicuticular dos biótipos de azevém resistente e suscetivel ao glyphosate; o biótipo resistente apresentou grau de polaridade pouco superior ao do biótipo suscetivel, porém essa diferença não pode ser considerada marcante a ponto de determinar maior ou menor tolerância de um biótipo ou outro ao herbicida glyphosate.
\end{abstract}

Palavras-chave: resistência, cromatografia, Lolium multiflorum, transgenia.

\begin{abstract}
The objective of this work was to determine the chemical composition of epicuticular wax of Italian ryegrass (Lolium multiflorum) biotypes resistant and susceptible to glyphosate, searching for relationships between their characteristics and the resistance of the biotypes to the product. The epicuticular wax was extracted and quantified, and its constituents analyzed by gas chromatography, coupled to mass spectrometer (CG-EM). To determine the chemical composition, leaf blade samples were obtained 30 days after plant emergence, the first leaf being collected with totally visible ligule. The amount of epicuticular wax did not differ between the biotypes. Alcohols are the most abundant among the compounds constituting the epiticular wax, represented by only one compound, hexacosan-1-ol (46.80\% in the resistant biotype and $52.20 \%$ in the susceptible). When comparing the polarity of the epicuticular wax of the Italian ryegrass biotypes, it was verified that both in the resistant and susceptible biotypes, the epicuticular wax presented more than $50 \%$ of polar compounds (alcohols and aldehydes) in its constitution, with that value being equal to 69.80\% in the resistant biotype and 64.94\% in the susceptible. Thus, it can be stated that small differences exist in the epicuticular wax of Italian ryegrass biotypes resistant and susceptible to glyphosate. The resistant biotype presented a slightly higher polarity degree than the susceptible biotype but such difference cannot be considered sufficiently relevant to determine a greater or smallertolerance of either biotype to glyphosate.
\end{abstract}

Keywords: resistance, chromatography, Lolium multiflorum, transgeny.

Recebido para publicação em 21.2.2008 e na forma revisada em 18.2.2009.

2 Mestranda do Dep. de Solos da UFLA, Caixa Postal 3037, 37200-000 Lavras-MG; ${ }^{3}$ Pós-Doutorado, Dep. de Fitotecnia da Universidade Federal de Viçosa - DFT/UFV, <evanderalves@yahoo.com.br>; ${ }^{4}$ Pesquisador da Embrapa Trigo, Passo Fundo-RS, <vargas@cnpt.embrapa.br>; ${ }^{5}$ Professor DFT/UFV; ${ }^{6}$ Doutorando do DFT/UFV; ${ }^{7}$ Professor do Dep. de Química - DEQ/UFV.

Planta Daninha, Viçosa-MG, v. 27, n. 1, p. 149-154, 2009 


\section{INTRODUÇÃO}

O azevém é uma planta anual, herbácea, sendo considerada importante planta daninha em culturas anuais e perenes na região Sul do Brasil, porém pode ser controlada facilmente (Roman et al., 2004; Vargas et al., 2005). Em decorrência do uso indiscriminado e repetitivo do glyphosate para controle dessa planta daninha na referida região, promoveu-se a seleção de biótipos resistentes a esse herbicida (Vargas et al., 2005), tornando o manejo extremamente dificil. Com o intuito de buscar alternativas para esse novo problema, foi necessário realizar estudos básicos sobre a biologia, fisiologia, química e capacidade competitiva entre esses diferentes biótipos de azevém suscetivel e resistente ao glyphosate (Ferreira et al., 2006, 2008).

A penetração de herbicidas pode ocorrer nas plantas pelos órgãos aéreos, como folhas, caules, flores e frutos, pelos órgãos subterrâneos, como raízes, rizomas, tubérculos, e também pelas sementes e, durante a germinação e a emergência, pela radícula e pelo caulículo (Silva et al., 2007). No entanto, são as folhas o principal órgão das plantas daninhas envolvido na penetração de herbicidas aplicados em pósemergência, pois a maior parte dos herbicidas comercializados atualmente é aplicada em pós-emergência (Procópio et al., 2003).

A composição química do revestimento cuticular é variada, sendo a cutina e a cera seus principais componentes. A cutina é um biopoliéster insolúvel, de elevado grau de entrecruzamento entre os ácidos graxos hidroxilados de cadeia longa que a compõem, enquanto a cera apresenta-se embebida nesse polímero ou depositada no exterior da cutícula (epicuticular). A cera epicuticular pode ser depositada em uma variedade de formas físicas: em algumas plantas, formam camadas finas; em outras, podem formar placas ou cristais de diferentes tamanhos e formas. Devido à sua composição química, refletida no grau de cristalidade, e à possibilidade de constituir na superficie da membrana cuticular uma película que atua como interfase entre a célula vegetal e o meio, a cera se destaca como a principal barreira protetora contra as perdas de água por transpiração excessiva, a ação de patógenos, as radiações solares e a entrada de produtos químicos e contaminantes (Heredia et al., 1998).

Dependendo da composição e da polaridade da cera e do herbicida, a cera epicuticular pode se tornar uma barreira à penetração de herbidas. A quantidade e a composição da cera epicuticular podem variar com a espécie, com o cultivar e até mesmo dentro da mesma população. As diferenças dependem, também, das condições climáticas e da fenologia da planta. A cera epicuticular é uma mistura complexa de diferentes compostos alifáticos. Normalmente, esses compostos contêm séries homólogas de alcanos, álcoois (primários e secundários), aldeídos, ácidos, cetonas, $\beta$-dicetonas e ésteres (Bianchi \& Bianchi, 1990).

Objetivou-se com este trabalho determinar a composição química da cera epicuticular dos biótipos de azevém resistente e suscetivel ao glyphosate.

\section{MATERIAL E MÉTODOS}

O experimento foi conduzido em câmara de crescimento com temperatura controlada (entre 18 e $22^{\circ} \mathrm{C}$ ), em vasos contendo $200 \mathrm{~mL}$ de substrato, composto de Argissolo VermelhoAmarelo adubado conforme análise de solo. Em cada vaso foram semeadas dez sementes de azevém, procedendo-se ao desbaste, mantendo duas plantas por vaso. O delineamento experimental foi o inteiramente casualizado, com dez repetições de cada biótipo de azevém (resistente e suscetivel ao glyphosate).

As folhas foram coletadas 30 dias após a emergência, e a cera epicuticular foi extraída da primeira folha com ligula totalmente visivel, utilizando-se a metodologia de Hamilton (1995), com modificações. Para isso, uma folha de cada repetição foi introduzida, separadamente, em placa de Petri com $100 \mathrm{~mL}$ de clorofórmio por 30 segundos, agitando-se levemente. Esse procedimento foi realizado cuidadosamente, para se evitar a ruptura dos tecidos foliares e a conseqüente liberação dos compostos celulares. Os extratos obtidos foram filtrados com papel-filtro e transferidos para um béquer, onde foram evaporados em banhomaria, até reduzir o volume para, aproximadamente, $15 \mathrm{~mL}$. Esta solução (clorofórmio mais cera) foi transferida para tubos de ensaio de $25 \mathrm{~mL}$, de peso conhecido. O clorofórmio foi 
evaporado em banho-maria, para obtenção do resíduo sólido (cera). A quantificação da cera foi expressa pela massa de cera por unidade de área foliar $\left(\mathrm{mg} \mathrm{cm}^{-2}\right)$. Para determinação da área das folhas de cada cultivar, utilizada para extração da cera, foi usado aparelho fotoelétrico de medição de área foliar (T Delta-T Devices).

As análises químicas foram realizadas no Laboratório de Análise e Sintese de Agroquímicos, pertencente ao Departamento de Química da Universidade Federal de Viçosa. Cada amostra foi submetida à análise em espectrômetro de infravermelho, registrado entre 4.000 e $500 \mathrm{~cm}^{-1}$.

As análises cromatográficas foram realizadas em aparelho GC-MS PQ 5050A (Shimadzu), utilizando-se coluna capilar DB-1 (30 m; 0,25 mm de diâmetro interno; filme de $0,25 \mu \mathrm{m})$ e hélio como gás de arraste. A temperatura do injetor foi de $290{ }^{\circ} \mathrm{C}$, com temperatura inicial de $80^{\circ} \mathrm{C}$, por cinco minutos, aumentando de 80 a $285^{\circ} \mathrm{C}$ na razão de $4{ }^{\circ} \mathrm{C} \mathrm{min}-1$. A temperatura final permaneceu em $285^{\circ} \mathrm{C}$ por 40 minutos. A temperatura do detector foi de $290{ }^{\circ} \mathrm{C}$, e a da interface do sistema CG-EM, de $290^{\circ} \mathrm{C}$. O detector de massas operou com ionização de chama por impacto de elétrons $(70 \mathrm{ev})$ e varredura de massas de 30 a $600 \mathrm{Da}$. Foi injetado $1 \mu \mathrm{L}$ de cada amostra (havia aproximadamente $10 \mu \mathrm{g}$ de cera epicuticular), e a identificação dos compostos foi feita por meio de comparação dos espectros de massa das amostras com aqueles existentes no banco de dados (Wiley 229.000).

Os dados referentes à quantidade de cera epicuticular foram submetidos à análise de variância e ao teste de Tukey a $5 \%$ de probabilidade. Para análise dos grupos de compostos químicos, utilizaram-se médias observadas e desvios-padrão.

\section{RESULTADOS E DISCUSSÃO}

A quantidade de cera epicuticular extraída (Figura 1) foi maior nas folhas do biótipo de azevém suscetivel $\left(16,20 \mu \mathrm{g} \mathrm{cm}^{-2}\right)$, porém sem diferir do biótipo resistente $\left(14,90 \mu \mathrm{g} \mathrm{cm}^{2}\right)$. A quantidade de ceras epicuticulares na maioria das espécies varia de 10 a $200 \mu \mathrm{g} \mathrm{cm}^{-2}$ (McWhorter \& Ouzts 1993), embora quantidades acima de $300 \mu \mathrm{g} \mathrm{cm}^{-2}$ já tenham sido relatadas (Baker, 1982). Ferreira et al. (2005), trabalhando com cinco genótipos de canade-açúcar, observaram que esses valores variavam de 16,26 a $23,22 \mu \mathrm{g} \mathrm{cm}^{-2}$, sem nenhuma relação com o grau de tolerância a herbicidas dos genótipos com a quantidade de cera. Monquero et al. (2004) observaram em Ipomea grandifolia $37,0 \mu \mathrm{g} \mathrm{cm}^{-2}$ de cera epicuticular, seguida por Commelina benghalensis $\left(34,5 \mu \mathrm{g} \mathrm{cm}^{-2}\right)$ e Amarantus hybridus $\left(31,7 \mu \mathrm{g} \mathrm{cm}^{-2}\right)$, também sem relação com absorção de herbicidas. Holloway (1970), Hodgson (1973) e Al-Jaff-Jaff et al. (1982) afirmam não existir correlação entre quantidade de cera epicuticular e absorção de herbicidas.

A cera epicuticular de cada material avaliado foi submetida à análise espectroscópica na região do infravermelho e caracterizada como uma mistura de compostos de cadeia curta. Essas observações são indicadas pelas bandas em 3.500-3.200 cm-2 (deformação de $\mathrm{O}-\mathrm{H}$ ), 2.910 e $2.810 \mathrm{~cm}^{-1}$ (deformação de $\mathrm{CH}_{2}$ e $\mathrm{CH}_{3}$ ), uma banda fica em $1.732 \mathrm{~cm}^{-1}$ (deformação $\mathrm{C}=\mathrm{O}$ de éster), $1.412-1.402 \mathrm{~cm}^{-1}$ (deformação $\mathrm{C}-\mathrm{H}$ ) e $720 \mathrm{~cm}^{-1}$ (deformação de $\left.\left[\mathrm{CH}_{2}\right]_{\mathrm{n}}, \mathrm{n}>4\right)$. O material presente nos biótipos (resistente e suscetivel) não mostrou diferenças pela análise de espectroscopia na região do infravermelho; por essa razão, apresentaram-se dados referentes ao biótipo resistente na Figura 2. Os compostos orgânicos presentes na mistura foram identificados por comparação com aqueles contidos na biblioteca do

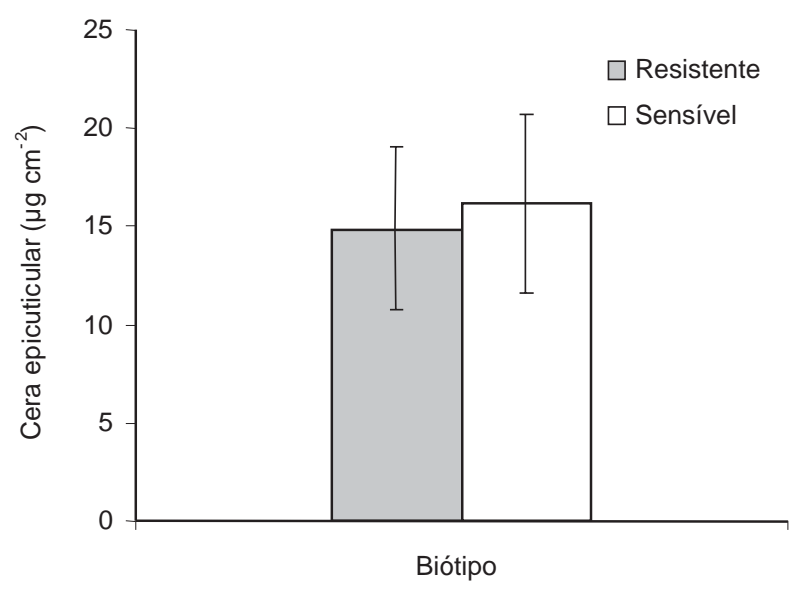

Figura 1 - Massa de cera epicuticular $\left(\mu \mathrm{g} \mathrm{cm}^{-2}\right)$ extraída de folhas de biótipos de azevém resistente e suscetível ao glyphosate. As barras representam o desvio-padrão. 


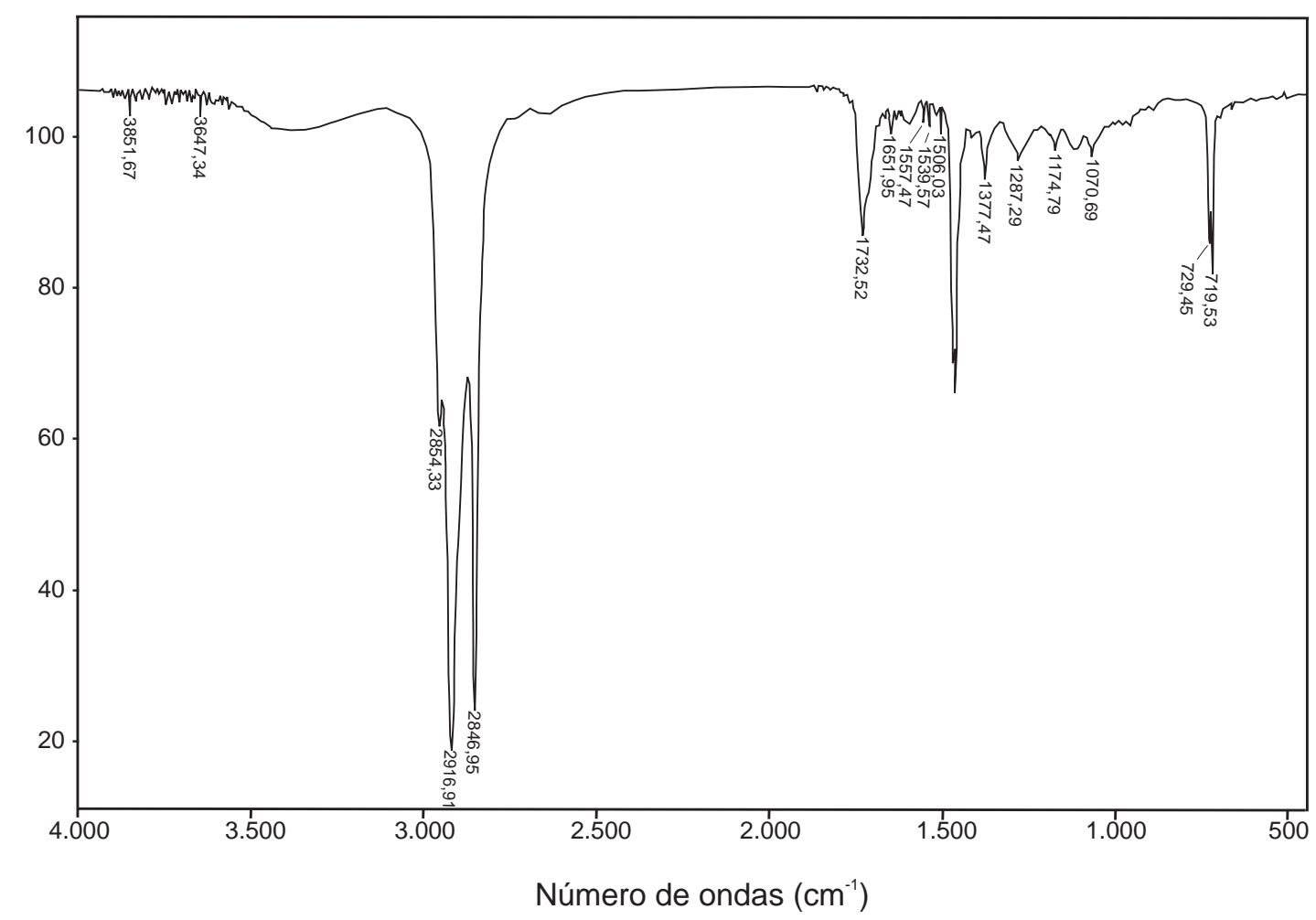

Figura 2 - Espectro no infravermelho $(\mathrm{NaCl})$ da fração da amostra da cera epicuticular do biótipo de azevém resistente ao glyphosate.

aparelho (Wiley 229.000). Os compostos identificados estão relacionados na Tabela 1 .

A cromatografia de fase gasosa indicou a existência de 20 a 40 constituintes na cera dos dois biótipos de azevém. No entanto, ao serem submetidos à espectrometria de massa, optou-se por incluir nos resultados aqueles com porcentagem igual ou superior a $1,60 \%$, sendo considerados dessa forma de 10 a 16 picos (Tabela 1).

Como pode ser observado na Tabela 1 , quatro compostos em cada um dos biótipos não puderam ser identificados. Os componentes enicosano, octadecanal e octadecano foram observados apenas no biótipo resistente de azevém, e o composto tricosano foi encontrado somente no biótipo suscetivel.

Os componentes tridecanal, hexacosan-1o1, hexadecanal, pentacosano e octacosano foram encontrados nos dois biótipos avaliados (Tabela 1).

Os grupos de compostos observados na cera epicuticular dos dois biótipos foram álcoois, aldeídos e hidrocarbonetos (Figura 2). Os hidrocarbonetos são os componentes menos polares (mais hidrofóbicos) encontrados na cera epicuticular dos dois biótipos de azevém. É conhecido o fato de que há uma interação bastante complexa entre a natureza química do produto aplicado e a superficie foliar. Existem dois tipos principais de superfícies: uma facilmente molhável (rica em álcoois) e outra de molhamento mais dificil (rica em alcanos). As características da solução aplicada, a polaridade do composto, a tensão superficial da calda etc. são importantes nessa interação (Silva et al., 2007). De acordo com Heredia et al. (1998), o que define a maior absorção de herbicidas pelas folhas das plantas é a composição da cera epicuticular. Herbicidas polares tendem a penetrar com maior facilidade em plantas onde as folhas apresentam constituição química da cera mais polar nesse caso, a cera epicuticular se hidrata com maior facilidade. Da mesma forma, herbicidas pouco polares penetram com maior facilidade em folhas que apresentam ceras formadas por compostos menos polares (hidrofóbicos). 
Tabela 1 - Composição química (\%) de cera epicuticular das folhas dos biótipos de azevém resistente e suscetível ao glyphosate

\begin{tabular}{|c|c|c|c|}
\hline \multirow{2}{*}{$\begin{array}{c}\text { TR } \\
\text { (minutos) }\end{array}$} & \multirow{2}{*}{ Composto } & \multicolumn{2}{|c|}{ Biótipo de azevém } \\
\cline { 3 - 4 } & & Resistente & Suscetível \\
\cline { 3 - 4 } & & \multicolumn{2}{|c|}{$(\%)$} \\
\hline 56,21 & Icosano & - & 1,96 \\
\hline 57,81 & N.I & - & 1,88 \\
\hline 58,81 & Enicosano & 1,58 & - \\
\hline 59,61 & Docosano & - & 2,23 \\
\hline 61,25 & N.I & 1,60 & 1,90 \\
\hline 62,08 & Tridecanal & 8,92 & 10,83 \\
\hline 62,65 & Tricosano & - & 1,88 \\
\hline 63,84 & Hexacosan-1-ol & 46,80 & 52,20 \\
\hline 65,46 & Tetracosano & 1,69 & 1,92 \\
\hline 65,45 & N.I & 1,62 & 1,93 \\
\hline 67,48 & Hexadecanal & 6,65 & 1,91 \\
\hline 68,16 & Pentacosano & 15,42 & 13,18 \\
\hline 70,90 & N.I & - & 4,67 \\
\hline 73,49 & Octacosano & 5,91 & 3,80 \\
\hline 71,53 & Octadecanal & 6,68 & - \\
\hline 77,96 & Octadecano & 3,09 & - \\
\hline
\end{tabular}

N.I - compostos não identificados; TR - tempo de retenção.

O composto observado em maior proporção nos biótipos resistente e suscetível de azevém foi o hexacosan-1-ol (46,80\% no biótipo resistente e $52,20 \%$ no biótipo suscetivel) (Tabela 1), sendo os álcoois os compostos mais abundantes observados na cera epicuticular dos biótipos de azevém, representados por apenas este composto, já mencionado. Aldeídos e álcoois são compostos polares de alta molhabilidade, o que facilita a penetração dos herbicidas polares, como o glyphosate (McWorter \& Ouzts, 1993). De modo geral, os álcoois são mais polares (hidrofilicos) que os hidrocarbonetos outro grupo de compostos encontrados na cera epicuticular dos biótipos de azevém.

Não se detectaram diferenças entre todas as classes de compostos representados na Tabela 2, tanto do biótipo de azevém resistente quanto do suscetivel. No entanto, Ferreira et al. (2006), também trabalhando com ${ }^{14} \mathrm{C}$ glyphosate, não observaram diferenças marcantes entre os dois biótipos de azevém com relação à absorção do glyphosate. Segundo
Hamilton (1995), os hidrocarbonetos estão presentes em quase toda a superficie lipídica das plantas, variando quantitativamente de traços até $50 \%$ da quantidade total, mas são predominantes em poucos casos. De acordo com Chachalis et al. (2001), os hidrocarbonetos de cadeia longa são menos polares que álcoois e ésteres. Monquero et al. (2004), trabalhando com as plantas daninhas Commelina benghalensis, Ipomoea grandifolia e Amaranthus hybridus, observaram que C. benghalensis, que possui maior tolerância ao glyphosate, apresentou maior proporção de hidrocarbonetos na cera epicuticular, altamente hidrofóbicos (pouco polares), contrariando a polaridade do glyphosate, que é altamente hidrofílico (polar) (Sandberg et al., 1980). Ferreira et al. (2005), trabalhando com cinco genótipos de cana-de-açúcar, constataram que a proporção desse composto na cera epicuticular variou de 5,76 a $18,16 \%$, porém sem nenhuma relação com absorção da mistura herbicida trifloxysulfuron + ametryn. Monquero et al. (2004) observaram que em C. benghalensis as ceras apresentaram em sua constituição química maior quantidade de hidrocarbonos (n-alcanos) em comparação com as espécies Ipomoea grandifolia e Amaranthus hybridus, constatando dessa forma relação entre a composição da cera epicuticular e a absorção do glyphosate.

Ao se comparar a polaridade da cera epicuticular dos biótipos de azevém, constatouse que tanto no biótipo resistente quanto no suscetivel a cera epicuticular apresentou mais de $50 \%$ de componentes polares (álcoois e aldeídos) em sua constituição, sendo esse

Tabela 2 - Grupos de compostos (\%) e desvio-padrão dos grupos de compostos encontrados na cera epicuticular das folhas dos biótipos de azevém resistente e suscetível ao glyphosate

\begin{tabular}{|l|c|c|}
\hline \multirow{2}{*}{ Composição } & Biótipo resistente & Biótipo suscetível \\
\cline { 2 - 3 } & \multicolumn{2}{|c|}{$(\%)$} \\
\hline Hidrocarbonetos & $27,69 \pm 11,0$ & $24,97 \pm 10,0$ \\
\hline Aldeídos & $22,25 \pm 10,0$ & $12,74 \pm 5,0$ \\
\hline Álcoois & $46,80 \pm 17,0$ & $52,20 \pm 23,0$ \\
\hline N.I & $3,22 \pm 3,0$ & $10,38 \pm 5,0$ \\
\hline
\end{tabular}

N.I - Compostos não identificados. 
valor igual a $69,80 \%$ no biótipo resistente e $64,94 \%$ no biótipo suscetível (Tabela 3). Informações referentes à polaridade da cera epicuticular estão disponiveis para várias espécies de plantas daninhas, sendo bastante variada. Como exemplo, pode-se citar Cyperus rotundus, que apresenta em sua constituição cerca de $82 \%$ de compostos não-polares, enquanto Sorghum halepense apresenta cerca de $6 \%$ de compostos polares e $93 \%$ da constituição química da sua cera epicuticular representada por compostos não-polares (Silva et al., 2007).

Tabela 3 - Porcentagem de compostos polares e pouco polares encontrados na cera epicuticular das folhas dos biótipos de azevém resistente e suscetível ao glyphosate

\begin{tabular}{|l|c|c|}
\hline \multirow{2}{*}{ Componente } & $\begin{array}{c}\text { Biótipo } \\
\text { resistente }\end{array}$ & $\begin{array}{c}\text { Biótipo } \\
\text { suscetível }\end{array}$ \\
\cline { 2 - 3 } & \multicolumn{2}{|c|}{$(\%)$} \\
\hline Polares (aldeídos + álcoois) & 69,80 & 64,94 \\
\hline Pouco polares (hidrocarbonetos) & 27,69 & 24,97 \\
\hline
\end{tabular}

Por meio dessa caracterização, pode-se afirmar que existem pequenas diferenças na composição da cera epicuticular dos biótipos de azevém resistente e suscetivel ao glyphosate, com diferenças também na polaridade da cera, que é ligeiramente maior no biótipo resistente. No entanto, é pouco provável que essa diferença seja suficiente para determinar o maior ou menor grau de resistência de um biótipo ou outro ao herbicida glyphosate.

\section{LITERATURA CITADA}

AL-JAFF, D. M. A. et al. Further studies on bracken morphology in relation to herbicide uptake. In: CUTLER, D F.; ALVIN, K. L.; PRICE, C. E. (Eds.). The plant cuticle. London: Academic Press, 1982. p. 293-301. (Linnean Society Symposium Series, 10)

BAKER, E. A. Chemistry and morphology of plant epicuticular waxes. In: CUTLER, D. F.; ALVIN, K. L.; PRICE, C. E. (Eds.). The plant cuticle. London: Academic Press. 1982. p. 139-166.

BIANCHI, A.; BIANCHI, G. Surface lipid composition of $\mathrm{C}_{3}$ and $\mathrm{C}_{4}$ plants. Biochem. Syst. Ecol., v. 18, n. 7-8, p. $533-537,1990$.

Planta Daninha, Viçosa-MG, v. 27, n. 1, p. 149-154, 2009
CHACHALIS, C. et al. Characterization of leaf surface, wax composition, and control of redvine and trumpet creeper with glyphosate. Weed Sci., v. 49, n. 1, p. 156-163, 2001.

FERREIRA, E. A. et al. Glyphosate translocation in Italian ryegrass biotypes (Lolium multiflorum). Planta Daninha, v. 24 , n. 2 , p. $365-370,2006$.

FERREIRA, E. A. et al. Sensibilidade de cultivares e clones de cana-de-açúcar à mistura trifloxysulfuron-sodium + ametryn. Planta Daninha, v. 23, n. 1, p. 93-99, 2005.

FERREIRA, E. A. et al. Distribuição de glyphosate e acúmulo de nutrientes em biótipos de azevém. Planta Daninha, v. 26, n. 1, p. 165-173, 2008.

HAMILTON, R. J. Waxes: chemistry, molecular biology and functions. Edinburgh: Orly Press, 1995. 149 p.

HEREDIA, A. et al. La cutícula vegetal: estructura y funciones. Ecologia, v. 12, n. 2, p. 293-305, 1998.

HODGSON, J. M. Lipid deposition on leaves of Canada thistle ecotypes. Weed Sci., v. 21, n. 1, p. 169-172, 1973.

HOLLOWAY, P. J. Surface factors affecting the wetting of leaves. Pestic. Sci., v. 1, n. 1, p. 156-163, 1970.

McWHORTER, C. G.; OUZTS, C. Leaf surface morphology of Erythroxylum sp. and droplet spread. Weed Sci., v. 12, n. 3, p. 293-305, 1993.

MONQUERO, P. A. et al. Caracterização da superficie foliar e das ceras epicuticulares em Commelina benghalesis, Ipomoea grandifolia e Amaranthus hybridus. Planta Daninha, v. 22, n. 2, p. 203-210, 2004.

PROCÓPIO, S. O. et al. Anatomia foliar de plantas daninhas do Brasil. Viçosa, MG: Universidade Federal de Viçosa, 2003. v. 1. 118 p.

ROMAN, E. S. et al. Resistência de azevém (Lolium multiflorum) ao herbicida glyphosate. Planta Daninha, v. 22 , n. 2, p. 301-306, 2004.

SANDBERG, C. L. et al. Absorption, translocation and metabolism of 14C-glyphosate in several weed species. Weed Res., v. 20, n. 1, p. 195-200, 1980.

SILVA, A. A. et al. Herbicidas: absorção, translocação, metabolismo, formulações e misturas. In: SILVA, A. A.; SILVA, J. F. (Eds). Tópicos em manejo de plantas daninhas. Viçosa, MG: Editora UFV, 2007. p. 149-188.

VARGAS, L. et al. Alteração das características biológicas dos biótipos de azevém (Lolium multiflorum) ocasionada pela resistência ao herbicida glyphosate. Planta Daninha, v. 23, n. 1, p. 153-160, 2005. 